University of Nebraska - Lincoln

DigitalCommons@University of Nebraska - Lincoln

\title{
Error analysis of large-eddy simulation of the turbulent non- premixed sydney bluff-body flame
}

\author{
A. M. Kempf \\ Imperial College London, Exhibition Road, South Kensington, London \\ B. J. Geurts \\ University of Twente, The Netherlands; Eindhoven University of Technology, The Netherlands \\ J. C, Oefelein \\ Sandia National Laboratories, Livermore, CA
}

Follow this and additional works at: https://digitalcommons.unl.edu/usdoepub

Part of the Bioresource and Agricultural Engineering Commons

Kempf, A. M.; Geurts, B. J.; and Oefelein, J. C, "Error analysis of large-eddy simulation of the turbulent non-premixed sydney bluff-body flame" (2011). US Department of Energy Publications. 116.

https://digitalcommons.unl.edu/usdoepub/116

This Article is brought to you for free and open access by the U.S. Department of Energy at DigitalCommons@University of Nebraska - Lincoln. It has been accepted for inclusion in US Department of Energy Publications by an authorized administrator of DigitalCommons@University of Nebraska - Lincoln. 


\title{
Error analysis of large-eddy simulation of the turbulent non-premixed sydney bluff-body flame
}

\author{
A.M. Kempf ${ }^{\text {a,* }}$, B.J. Geurts ${ }^{\text {b,c }}$, J.C. Oefelein ${ }^{\mathrm{d}}$ \\ ${ }^{a}$ Department of Mechanical Engineering, Imperial College London, Exhibition Road, South Kensington, London SW7 2AZ, United Kingdom \\ ${ }^{\mathrm{b}}$ Multiscale Modeling and Simulation, Faculty EEMCS, University of Twente, P.O. Box 217, 7500 AE Enschede, The Netherlands \\ ${ }^{\mathrm{c}}$ Anisotropic Turbulence, Laboratory for Fluid Dynamics, Faculty Applied Physics, Eindhoven University of Technology, P.O. Box 513, 5600 MB Eindhoven, The Netherlands \\ ${ }^{\mathrm{d}}$ Combustion Research Facility, Sandia National Laboratories, P.O. Box 969, Livermore, CA 94551, USA
}

\section{A R T I C L E I N F O}

\section{Article history:}

Received 18 June 2010

Received in revised form 5 March 2011

Accepted 18 April 2011

Available online 14 May 2011

\section{Keywords:}

Large-eddy simulation

Turbulent non-premixed combustion

Error analysis

\begin{abstract}
A B S T R A C T
A computational error analysis is applied to the large-eddy simulation of the turbulent non-premixed Sydney bluff-body flame, where the error is defined with respect to experimental data. The errorlandscape approach is extended to heterogeneous compressible turbulence, which is coupled to combustion as described by a flamelet model. The Smagorinsky model formulation is used to model the unknown turbulent stresses. We introduce several measures to quantify the total simulation error and observe a striking 'valley-structure' in the error that arises as function of the spatial resolution and the Smagorinsky length parameter. The optimal refinement strategy that can be extracted from this error-landscape is reminiscent of that for non-reacting turbulent flow.
\end{abstract}

ㄷ 2011 The Combustion Institute. Published by Elsevier Inc. All rights reserved.

\section{Introduction}

Robust and efficient combustor designs that are suitable for a wide range of fuels are becoming of key importance with declining resources, rising fuel prices and growing concerns about carbon emissions. The industrial design of such advanced combustion systems employs more and more the capabilities of computational modeling to investigate future improvements. However, turbulent combustion presents major challenges to computational modeling, which currently restricts the use of computational design strategies. Compared to the turbulent flow of a simple fluid such as air, combustion considerably increases the complexity of the problem. A particular challenge arises from the highly localized regions of intense combustion and the rapid response of some of the chemical processes that take place. Compared to length and time-scales that are characteristic of small-scale single phase turbulence, both the spatial localization and the time-scales of combustion are much smaller and much more rapid respectively. Under realistic flow and process conditions, the resulting complexity is too large to allow a full resolution with modern supercomputers. This has sparked a variety of modeling strategies, addressing (i) the dynamic consequences of small-scale turbulent motions, (ii) the resulting multitude of chemical transformations that constitute the total combustion, and (iii) the complete coupling between these non-linear processes.

\footnotetext{
* Corresponding author.

E-mail address: a.kempf@imperial.ac.uk (A.M. Kempf).
}

We use the concept of large-eddy simulation (LES) that is based on the spatial filtering of the governing equations. The origin of the LES technique is normally seen in weather forecasting [1], where a time-resolved prediction is inherently required. The LES approach has been extended to engineering applications, and is developed for complex systems involving multi-phase flow, chemical reactions, real-gas effects, and variable density [2-4]. The present paper focuses on non-premixed combustion, where fuel and oxidiser only meet in the combustion chamber, and the rate of chemical reaction is limited by the rate of mixing. Cook and Riley [5] used a priori analysis to develop a subgrid model with equilibrium chemistry, which was later applied by Branley and Jones [6] in a real, fully coupled LES. Forkel developed an efficient method for the LES of diffusion flames based on the mixture fraction approach [7], which was later extended [8] to a steady flamelet description $[9,10]$. Pitsch further improved the prediction of minor species by considering unsteady flamelets [11]. Navarro-Martinez et al. [12] applied Bilger and Klimenko's full [13] CMC (Conditional Moment Closure) technique to LES, after the CME (Conditional Moment Estimation) approach had already been tested by Steiner and Bushe [14]. To consider more detailed chemistry and scalar mixing, various methods for transporting a filtered density function were developed and tested by Colucci et al. [15], Kerstein et al. and Menon et al. [16,17], Raman et al. [18], Bisetti et al. [19], and Mustata et al. [20]. Further details on contributions to the non-premixed combustion LES can be found in the reviews presented by Vervisch and Poinsot [21], Janicka [22], Oefelein [23] and Pitsch [24]. We adhere to modeling strategies that follow the spatial filtering 
approach that is basic to large-eddy simulation. Consequently, we include sub-filter modeling of the turbulent stresses and a reduced order flamelet representation of the combustion. Such modeling is aimed at reducing the complexity of the underlying dynamical system to allow simulation at a strongly reduced spatial resolution, i.e., at manageable computational costs. The ability of LES to (conceptually) accommodate a wide range of flow coarsening to minimize the computational effort is also its main drawback. Little quantitative information is available that defines just how much coarsening is allowable as a function of accuracy. Hence, an unexpecting practitioner of LES may inadvertantly exaggerate the flow coarsening to minimize the cost, with the risk of relying too much on the mathematical-physical modeling of the small-scale motions and inherent errors. Likewise, the effects of competing numerical errors on the physical models must also be considered. It is essential that a systematic assessment of the reliability of LES becomes available that facilitates the use of LES in modern design processes.

In this paper we elaborate and further develop the errorlandscape [25-27] approach to quantify the simulation error as function of spatial resolution and sub-filter model parameters. We observe that the striking valley-structure of the total simulation error found for homogeneous isotropic decaying turbulence, also occurs in our model of turbulent, non-premixed combustion. This signifies that the phenomenon of partial cancellation of modeling and discretization errors in standard LES $[3,28]$ also plays a major role for turbulent combustion. The valley-structure in the error-landscape is hence also observed in flows that are spatially heterogeneous and include modulation of turbulence through the coupling with non-premixed combustion. This extension of the error-landscape framework to reacting flows suggests that the valley-structure may be a generic feature for a wider range of complex flow problems, suggesting new computational strategies for optimal simulation at pre-specified spatial resolution [29].

Currently, much industrial flow analysis is based on the wellestablished Reynolds averaged Navier-Stokes approach (RaNS). The closure of the turbulent stresses in RaNS involves reference scales that are characteristic of the large scales, e.g., scales associated with the mean flow. The RaNS approach requires a computational effort that can quite readily be supplied by currently available infrastructure. As a result, it is now common practice to resolve RaNS models to such a degree of detail that the solution can be considered as spatially converged, leaving only an uncertainty in the quality of the final solution arising from deficiencies in the turbulence model. In LES the error structure is considerably more complex. This arises from the fact that in most practical LES the spatial resolution of the sub-filter scales is (virtually) absent. In fact, the filter-width $\Delta$ and the mesh-spacing $h$ are often taken to be equal, or have a ratio $\Delta / h=2$. This implies that a significant contribution to the total simulation error is due to the numerical method. Moreover, this numerical contribution will interact with errors due to deficiencies in the mathematical-physical modeling of the small-scale phenomena, which in general may lead to a non-linear accumulation in which errors can partially cancel under certain numerical conditions, while errors may amplify monotonously at other numerical conditions. This yields an essential difference in the LES error dynamics compared to RaNS, and requires a new approach to error assessment.

The topic of reliability of LES has gained considerable attention in the past decade. Early studies include the work of Ghosal [30] and Vreman et al. [31,28], exemplifying non-linear accumulation of errors in simulations of turbulence. Through a judicious combination of fully resolved direct numerical simulation and large-eddy simulation at a fixed filter-width $\Delta$ it is possible to decompose the total error into its contributing modeling and discretization errors. For that academic purpose a combination needs to be made of a practical LES and a grid-independent LES at sufficiently large
$r=\Delta / h$. In practice a value $r \geqslant 4$ was found to be adequate for mean and rms properties of turbulent solutions [32]. The decomposition of the total error in these components revealed that the non-linear accumulation may lead to partial cancellation for some flow properties, which seriously complicates the error assessment. In later years, the computational analysis of the LES error was extended to a first systematic parameter study in which the limitations in the accuracy due to modeling and discretization were correlated with the 'subgrid activity' parameter $s$ [32]. This parameter $s$ was defined as the ratio of the subgrid dissipation relative to the total dissipation. Low values of $s$ implied that discretization errors were dominant while values of $s$ close to unity suggest that the subgrid modeling errors are dominant. The work of Meyers et al. [25] extended the parameter study and recorded the first systematic error-landscapes for a Smagorinsky [1] subgrid model, in which the total simulation error was determined at a large range of resolutions $N$ and subgrid model parameter $C_{S}$. A striking 'valley-structure' was observed in the total simulation error based on the resolved kinetic energy or a generalization in which different weights were assigned to different wavenumber bands [27]. From a physical point of view, a combination of error-measures that are sensitive to large scales (integral length-scale), intermediate scales (resolved kinetic energy) and small-scales (resolved enstrophy) was found to yield a robust impression of the limiting errors. An ultimate consequence of the recorded valley-structure was exploited by Geurts and Meyers [29] by determining algorithmically the conditions of optimal error-cancellation at fixed spatial resolution. Albeit ad hoc, this optimization was shown to yield reductions in error levels of up to about one decade in some cases. The work on the issue of reliability of LES has shown that it is much more difficult to quantify the error in a certain simulation, than it is to achieve a first simulation of flow in a certain configuration.

This paper applies the error-landscape approach to the Sydney bluff-body flame [?,33], implying that previous work on LES errors is extended to spatially heterogeneous turbulence. The resulting error-landscape was found to feature a global optimum for $C_{S}=0.13$ on the finest grid, leading to good predictions of the flow, mixing and combustion. The error-landscape is smooth near its optimum, implying a low sensitivity to grid and model parameter variations. A non-gradient based optimization technique (SIPI [29]) for optimizing the model constant was demonstrated in the vicinity of the global optimum. This paper is organized as follows. In Section 2 we introduce the LES approach to the Sydney flame and describe in detail the flamelet model that was adopted. Section 3 is devoted to the introduction of the systematic error-landscape approach for quantifying the total simulation error that occurs. A review of errormeasures is provided in this section. Subsequently, in Section 4 the simulation results are collected while the structure of the errorlandscape is presented in Section 5. Attention is given to the generic aspects of the error-landscape, its robustness with respect to the error-measure that was adopted and the implications for achieving optimal cancellation of modeling and discretization errors. Finally, concluding remarks are collected in Section 6.

\section{Large-eddy simulation with steady flamelet chemistry}

In this section we first introduce the mathematical model used to simulate the turbulent non-premixed Sydney bluff-body flame (Subsection 2.1) and subsequently describe the numerical method used to discretize the equations (Subsection 2.2).

\subsection{Equations and modeling}

To simulate turbulent non-premixed combustion, the spatially filtered Navier-Stokes Eq. (1) are solved together with the 
continuity Eq. (2) at low Mach numbers. We employ a convolution filter, which commutates with partial derivatives and find:

$\frac{\partial}{\partial t}\left(\bar{\rho} \tilde{u}_{i}\right)+\frac{\partial}{\partial x_{j}}\left(\bar{\rho} \widetilde{u_{i} u_{j}}\right)=$

$\frac{\partial}{\partial x_{j}}\left[\bar{\rho} \tilde{v}\left(\frac{\partial \tilde{u}_{j}}{\partial x_{i}}+\frac{\partial \tilde{u}_{i}}{\partial x_{j}}-\frac{2}{3} \bar{\rho} \tilde{v} \frac{\partial \tilde{u}_{k}}{\partial x_{k}} \delta_{i j}\right)\right]-\frac{\partial \bar{p}}{\partial x_{i}} \frac{\partial \bar{\rho}}{\partial t}+\frac{\partial \bar{\rho} \tilde{u}_{j}}{\partial x_{j}}=0$

Here, $t$ denotes time and $x_{i}$ the ith Cartesian coordinate. The density is given by $\rho$, the velocity in the $x_{i}$-direction by $u_{i}$ and $p$ represents the pressure. The filtered kinematic viscosity is represented by $\tilde{v}-$ it is assumed to be a function of temperature $T$ and to a smaller extend of chemical composition. The spatial filtering is denoted by an overbar over a variable. In addition, we distinguish variables to which density weighted filtering was adopted; so-called Favre filtering where $\widetilde{\Phi}=\overline{\rho \Phi} / \bar{\rho}$. The flow dynamics that follows from these equations is affected by the heat release coming from the combustion process that takes place in the turbulent flow. In the low Mach number approximation a degree of "incompressibility" arises where the density $\rho$ remains variable but independent of the pressure $p$.

The momentum equation contains unknown correlations of velocities and viscosity. The velocity products $\widetilde{u_{i} u_{j}}$ are described by the products of resolved velocities $\tilde{u}_{i} \tilde{u}_{j}$ and the subgrid stresses due to the unresolved turbulent motions $\tau_{i j}^{\text {sgs }}$ according to $\tau_{i j}^{s g s}=\tilde{u}_{i} \tilde{u}_{j}-\tilde{u}_{i} \tilde{u}_{j}$. The viscous fluxes formally also induce a closure problem as $v(T) \neq v(\widetilde{T})$. However, the difference between the leftand right-hand side are normally ignored at low Mach numbers [37], leading to the following approximation of the momentum equation:

$\frac{\partial}{\partial t}\left(\bar{\rho} \tilde{u}_{i}\right)+\frac{\partial}{\partial x_{j}}\left(\bar{\rho} \tilde{u}_{i} \tilde{u}_{j}\right)=$

$\frac{\partial}{\partial x_{j}}\left[\bar{\rho} \tilde{v}\left(\frac{\partial \tilde{u}_{j}}{\partial x_{i}}+\frac{\partial \tilde{u}_{i}}{\partial x_{j}}\right)-\frac{2}{3} \bar{\rho} \tilde{v} \frac{\partial \tilde{u}_{k}}{\partial x_{k}} \delta_{i j}+\bar{\rho} \tau_{i j}^{s g s}\right]-\frac{\partial \bar{p}}{\partial x_{i}}$

Here the molecular viscosity $\tilde{v}$ is a function of temperature and gascomposition and is obtained from the flamelet chemistry model $[38,39]$ that will be described in a later paragraph. The unknown subgrid stresses $\tau_{i j}^{s g s}$ must be modeled. For this problem various suggestions have been put forward in literature [2,3]. Here we will adopt a classical eddy-viscosity model in which we put:

$\tau_{i j}^{s g s}=v_{t}\left(\frac{\partial \tilde{u}_{j}}{\partial x_{i}}+\frac{\partial \tilde{u}_{i}}{\partial x_{j}}-\frac{2}{3} \frac{\partial \tilde{u}_{k}}{\partial x_{k}} \delta_{i j}\right)$

This is known as the gradient hypothesis. To specify this model we require the turbulent viscosity $v_{t}$ for which we adopt the wellknown Smagorinsky model [1]. Smagorinsky's idea from 1963 was to calculate the turbulent viscosity from the deformation rate tensor. The magnitude of this dissipative flux is governed by the size of the strain-rate tensor and the model parameter $C_{S} \Delta$, which is the product of a non-dimensional coefficient $C_{S}$ and the LES filterwidth $\Delta$. The turbulent eddy-viscosity in Smagorinsky's model is given by Eq. (5), where the compressible contribution to the strain tensor was neglected:

$v_{t}=\left(C_{S} \Delta\right)^{2} \sqrt{2 \widetilde{S}_{i j} \widetilde{S}_{i j}} ; \widetilde{S}_{i j}=\frac{1}{2}\left(\frac{\partial \tilde{u}_{j}}{\partial x_{i}}+\frac{\partial \tilde{u}_{i}}{\partial x_{j}}\right)$

The Smagorinsky model is specified in terms of the product $C_{S} \Delta$. This subgrid model corresponds to a spatial filter with which the flow was smoothed. In addition, the spatial discretization method will induce an 'implicit filter' [40] in which the grid-spacing is the relevant length-scale. Actual LES is hence associated with both the explicit filtering (represented by the explicit subgrid model) and implicit filtering (associated with the spatial discretization). Here, we take the filter-width $\Delta$ equal to the grid-spacing and concentrate on effects due to variations in $C_{S}$ alone. The model parameter
$C_{S}$ is varied over a large range from a value slightly less than 0.065 as suggested by Piomelli [41] to up to twice Lilly's theoretical value of $C_{S 0}=0.173$ [42] - this represents a range of almost an order of magnitude considering that the model is based on the square of $C_{S} \Delta$. Various dynamic extensions of Smagorinsky's model have been proposed $[43,44]$ to automatically determine a suitable value for the model constant. Here we stick to the static version and extend the proposed error-landscape framework [25] to a spatially heterogeneous combustion flow problem.

Previous work showed that there is an optimal value for the Smagorinsky parameter $C_{S}$ that depends on a number of aspects of the flow simulation. In fact, the optimal value was found to depend on several aspects such as the flow-conditions, the spatial resolution and the numerical method, among others [27]. This was investigated in detail for homogeneous, isotropic decaying turbulence. The optimal value for $C_{S}$ corresponds to settings that balance a partial cancellation of errors due to modeling and numerical method [45]. For small $C_{S}$, the numerical error becomes dominant. For sufficiently large $C_{S}$ too much energy is removed from the solution which corresponds to situations in which the modeling error is dominant. Changing the parameter $C_{S}$ at a fixed spatial resolution, gives some control over the relative importance of errors due to subgrid modeling and numerical discretization. Correspondingly, the presence and accuracy of the smaller turbulent scales in numerical solutions can be influenced as is illustrated in the scalar fields shown in Fig. 1. We observe that at $C_{S}=2 C_{S 0}$ a snapshot of the temperature field conveys a rather smooth flow. At much lower values, e.g., $C_{S}=C_{S 0} / 4$ a lively numerical solution is obtained, however, without any guarantee whether or not the predicted small turbulent scales are corresponding to actual flow structures. In order to quantify the total simulation error and hence establish the reliability of individual simulations we proceed in the next section with a detailed error-landscape analysis in which the solutions at a range of $C_{S}$ values and various spatial resolutions will be compared with experimental data.

Non-premixed combustion (at high Damköhler numbers) is mixing limited, which means that fuel and oxidiser can be assumed to react as soon as they come together, since the mixing takes so much longer than the reaction. In this work, combustion is modeled using a flamelet approach according to Williams [9] and Peters [10], based on Bilger's mixture fraction [46]. The thermo-chemical state is then calculated from a look-up table that stores density, viscosity, temperature and species-concentrations as a function

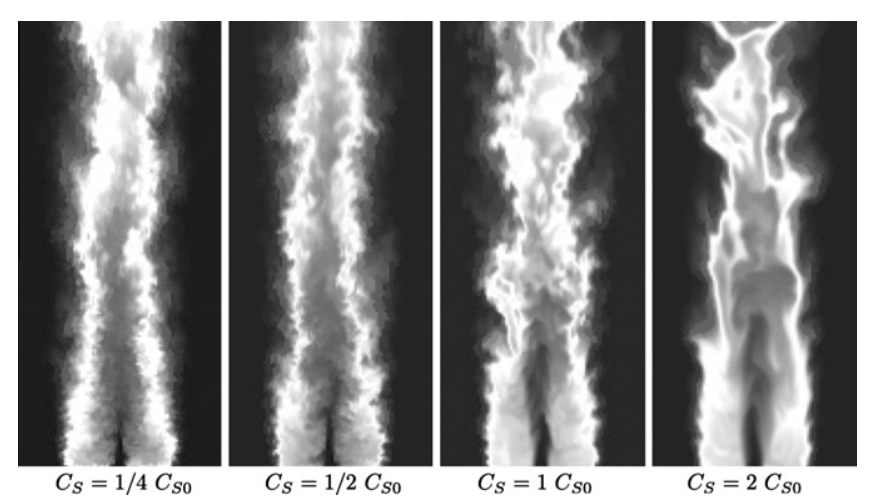

Fig. 1. Instantaneous temperature fields of the Sydney bluff-body flame for four different model constants relative to Lilly's suggestion of $C_{S O}=0.173$, using a grid of $5 \mathrm{M}$ cells. The figure shows how larger model constants suppress the smallest structures through artificial diffusion. With the smallest model constant, cell-sized structures which induce large numerical errors become dominant. As a result of interacting errors (and perhaps counter-intuitively), the flame front appears thicker for a very low Smagorinsky constant of $1 / 4 C_{S 0}$ than for the more realistic, higher values. 
of the mixture fraction field, using the procedure presented in [38]. In the present work, a single laminar flamelet was deemed sufficient as for a stably burning flame, the temperatures are (almost) independent of the strain-rate. The subgrid distribution of the mixture fraction is modeled by a top-hat function and an algebraic variance model, that have been shown to be more accurate for LES than the more complicated $\beta$-function [47]. The flamelet tables were provided by Lindstedt and co-workers, based on comprehensive, detailed chemistry [39].

\subsection{Discretisation and implementation}

We apply the PsiPhi LES code [48,49] for general transported scalars $\Phi$ and derived quantities $\Psi$. This code is based on the algorithms applied in the flowsi code developed at TU-Munich, TUDarmstadt and Imperial College [7,50,51], but PsiPhi makes heavy use of modern FORTRAN features such as the compact array-syntax, to speed up development, deployment, and run-time. The code uses a low Mach assumption to solve the Favre filtered NavierStokes equations on a collocated Cartesian grid which consists of cubic cells. The momentum subgrid fluxes are modeled using Smagorinsky's model [1]. The PsiPhi code applies a low storage RungeKutta scheme that is third order accurate for linear problems. For the non-linear convection, PsiPhi provides a range of methods, including upwind, quadratic upwind, central (2nd and 4th order) and TVD schemes. For this work, convection of momentum was described by the 2nd order central scheme, which has the lowest cost of the available schemes. The central scheme can only be used for the transport of momentum, where the continuity equation inhibits numerical oscillations. For scalar transport, numerical oscillations must be prevented through non-oscillatory schemes. We employ a TVD (Total Variation Diminuishing) scheme that blends a second order central scheme with a first order upwind scheme in a way that combines good accuracy with limited numerical oscillation. Avoiding numerical oscillation is particularly important for reacting scalars (here the mixture fraction), as the density depends on these in a strongly non-linear manner. To obtain realistic turbulent inflow conditions, artificial turbulence was created [52] to reproduce the experimental data at the most upstream location.

\section{Error landscape}

In this section we will introduce the error-landscape approach with which the error analysis of the Sydney bluff-body flame will be executed. A central component of the error analysis is the definition of the error-measure - here we will confront simulation results with available experimental data [33-35,53,54]. The basic structure of the error-landscape will be sketched afterwards in combination with the possibility for computational errorreduction.

The filtering approach to LES provides a theoretical motivation for introducing a model for the contribution of the sub-filter scales to the dynamics of the resolved scales. A large variety of such models has been suggested in literature [3], all aiming to reduce the computational complexity of the problem by reducing the contribution of the smallest scales. While this is beneficial for reducing the computational resources that are required, it is also a source of error in a simulation. The sub-filter model represents an approximation of the actual contribution from the dynamics at the smallest scales. This we will refer to as the modeling error; for a selected sub-filter model it will of course depend on the value of the parameters that define the model. In case of the Smagorinsky model this is the value of $C_{S}$ that enters the description. While in earlier literature values for this parameter were derived on the basis of assumed properties of homogeneous, isotropic turbulence, it is more common to select values for $C_{S}$ that are specific to a flow problem. Taking this one step further, we may consider $C_{S}$ a parameter with which one may optimize a given LES strategy.

The numerical treatment of LES constitutes a source of errors. Its importance depends on the spatial resolution relative to the retained smallest scales in the LES. By the introduction of a subfilter model, one has also introduced a new length-scale into the simulation, i.e., the filter-width $\Delta$. Many practitoners of LES use computational grids with mesh-spacing $h$ equal to the filter-width $\Delta$. At such marginal subgrid-resolution $r=\Delta / h$, scales on the order $\Delta$ are surely not well represented numerically. This opens the possibility of generating considerably contaminated dynamics for the resolved scales of order $\Delta$. This contamination will be specific to the numerical method that is adopted. Theoretically, if one allows $r$ to be sufficiently large, it is possible to achieve a grid-independent LES. It was found for flow in a turbulent mixing layer that $r \geqslant 4-6$ is adequate to capture mean and fluctuating properties using second or fourth order accurate finite volume discretizations [32]. Any more practical sub-filter resolution at $r=1$ or $r=2$ will require much less computational costs, but at the same time will have a stronger risk of small-scale contamination. This we will refer to as the discretization error.

In any large-eddy simulation the numerical flow prediction will be influenced by the interplay between sources of error arising from flaws in the sub-filter model and errors due to the numerical treatment at marginal sub-filter resolution. For strongly turbulent flow it is unclear how these sources of error will accumulate. This introduces the issue of reliability of a given LES. Since the effects of the sources of error may partially counteract for various flow properties, the resolution to the reliability issue is not simply to adopt the 'best' sub-filter model in combination with the highest order numerical discretization. This is all the more true in case the mesh-spacing is taken about equal to the filter-width as is often the case in practical LES applications. For this purpose the so-called error-landscape approach was introduced, providing a framework for systematically assessing the total simulation error that results from the combination of specific models and numerical methods in any given flow. It was introduced in the context of homogeneous, decaying turbulence and an extensive study was made of the influence of the flow-conditions and the numerical method on the total error behavior [25].

For the Smagorinsky model as adopted in this paper, a detailed impression of the total error behavior in a given flow may be obtained by considering the total error as a function of the numerical resolution $N \sim 1 / h$ and the resolution of the Smagorinsky length $C_{S} \Delta / h$. In this paper we will take $\Delta=h$. Any simulation in particular is then characterized by its combination $\left(C_{S}, N\right)$. Correspondingly, one may label the total error $E$ in this way. From contour-plots of $E$ as function of $C_{S}$ and $N$ one may infer under what conditions on the model parameter and/or the numerical resolution one may expect a given level of error. Such a contour-plot of $E\left(C_{S}, N\right)$ will be referred to as an error-landscape. It requires a systematic collection of individual LES on a grid of $\left(C_{S}, N\right)$ points. From such a study one may, afterwards, identify the 'optimal refinement' strategy, which specifies the value of $\widehat{C}_{S}$ at which the total error is minimal at given $N$.

A crucial element in the error-landscape approach is the measure that is adopted to quantify the total error. This aspect is dependent on the particular flow and application that is being investigated. In the study of the error behavior in homogeneous, isotropic, decaying turbulence [25] the choice was made to compare the LES predictions to filtered DNS results. The comparison included errors with different sensitivities to the larger or the smaller resolved scales, simultaneously. This was achieved by including predictions for, e.g., the resolved kinetic energy, but also 
the resolved enstrophy and dissipation. This weighing of components of the total error due to different scales in the flow, contributes to the robustness of predictions of the error behavior and allows to identify an optimal refinement strategy that is likely to be also 'near-optimal' for other flow properties.

In most simulation studies, no reference DNS data are available to assess the interacting error dynamics in a class of LES. In such cases one has to rely on case-specific information. This may include knowledge of the asymptotic behavior in certain limits or the inclusion of available experimental observations. In this paper we adopt the latter option and investigate the total simulation error in terms of differences that occur in mean and root-mean-square properties of the flow, such as velocity components, temperature and mixture fraction. For the Sydney bluff-body flame these data have been recorded experimentally for a variety of operating conditions, thereby providing a unique opportunity to extend the error-landscape approach to more realistic problems.The experimental data were recorded as mean and root-means-squares at different positions $\mathbf{x}_{i}$ at various sampling times $t_{n}$. These mean and RMS values $\xi_{\alpha, i}$ were obtained from quantities such as the velocity components, mixture fraction or temperature. The experimental $\xi_{\alpha, i}^{(\exp )}$ are compared directly to the respective means and RMS values $\xi_{\alpha, i}^{(\mathrm{LES})}$ of the LES predicted filtered flow quantities using an error definition

$E^{2}=\sum_{\alpha=1}^{A} \sum_{i=1}^{M} W_{\alpha, i}\left(\xi_{\alpha, i}^{(L E S)}-\xi_{\alpha, i}^{(\exp )}\right)^{2}$

for $A$ quantities to be recorded at $M$ locations.

This means that in the end, we compare Reynolds- or Favreaverages from experiment and simulation. Additional weighing was introduced through $W_{\alpha, i} \geqslant 0$. This general setting allows a number of more particular uses, of which we will consider:

- Single quantity: By setting $W_{\alpha, i}=0$ for all $\alpha$ apart from $\hat{\alpha}$ one may zoom into the error behavior of a particular flow property.

- Relative error weighing: When more than one flow property is included simultaneously in the analysis it appears best to include contributions that are properly scaled to only reflect the relative magnitude and importance of the error in a certain quantity.

Many more choices for the error weighing can be motivated for particular purposes - here we will not elaborate on this but consider a possible additional use of an error-landscape next.

The complete registration of a section of an error-landscape can be rather time consuming. For the strict error-minimisation at given resolution this is not needed; a more direct minimisation suggests itself. In addition, once an error-landscape has been recorded for a particular case, one may hypothesise that its structure may be similar for 'nearby' flow problems, e.g., differing in Reynolds number or combustion conditions. If one can extend the definition of the error-measure to approximate the total error under these 'slightly' different conditions it becomes possible to determine the optimal Smagorinsky parameter at given spatial resolution at strongly reduced computational overhead. Rather than computing a full error-landscape it then suffices to directly minimize the total simulation error. This optimization was first proposed in [29] in which Successive Inverse Polynomial Interpolation (SIPI) [55] was used to efficiently approximate the optimal $\widehat{C}_{S}$ at given $N$.

To approximate the lowest total simulation error for the Smagorinsky fluid at given spatial resolution $N^{3}$ we may iteratively adapt the Smagorinsky parameter. The first task is to obtain a so-called 'bracketing' interval $[a, c]$ which contains the optimal $\widehat{C}_{s}$. As first point of reference we use a 'no-model' simulation: $C_{S}=0=a$. This characterises the effects of discretization error only, but is not sta- ble with most numerical schemes. A second point of reference is obtained by using a 'practical' upper-bound $C_{S}=0.35$. This value of $C_{S}$ was found in a variety of literature to yield Smagorinsky LES predictions that are (much) too dissipative. Any further increase of $C_{S}$ above this practical upper-bound would most likely only add to the total error. This provides a practical upper-bound for the desired interval.

In view of the high computational effort that is required to evaluate $E$ only minimisation algorithms that do not rely on derivatives of $E$ appear suitable [55]. Locally around its minimum $E\left(C_{S}\right)$ at given $N$ can be approximated by a parabola in $C_{S}$. We start the iterations with an LES at the mid-point $C_{S}=b=c / 2$. Referring to Fig. 2, we then construct an interpolating parabola through $(a, E(a)),(b, E(b))$ and $(c, E(c))$. The minimum of this parabola is at

$d=b-\frac{1}{2} \frac{(b-a)^{2}[E(b)-E(c)]-(b-c)^{2}[E(b)-E(a)]}{(b-a)[E(b)-E(c)]-(b-c)[E(b)-E(a)]}$

provided the three points are not collinear. After a large-eddy simulation at $C_{S}=d$ the corresponding simulation error can be evaluated, a new triplet may be identified and the process may be continued. If we start from a 'proper' bracketing interval in which $E(b) \leqslant E(a)$ and $E(b) \leqslant E(c)$ then it is guaranteed that the new iterand $d \in] a, c[$. In case we start from an improper bracketing interval in which $E(b) \neq \min \{E(a), E(b), E(c)\}$ the new location may be above the upper bracket, $d>c$. However, since the error $E\left(C_{S}\right)$ is expected to increase monotonously for large $C_{S}$ this wider interval may be more suitable for further iteration. Subsequently, the iteration process converges via a sequence of proper bracketing intervals. This method of error-minimisation was adopted to homogeneous, isotropic, decaying turbulence [29] and to turbulent channel flow and a temporal mixing layer [56]. It was found that after about 46 iterations, involving separate large-eddy simulations, the optimal Smagorinsky coefficient is well approximated.

The computational overhead of the SIPI optimization is considerable but often well justified by the reduced error level. This may be quantified by incorporating the resolution dependence of the CPU time $T \sim N^{4}$. As an example, compared to the costs at $N=32$, a simulation at $N=48$ is about $(48 / 32)^{4}=5$ times more expensive while $N=64$ requires $(64 / 32)^{4}=16$ times more effort. If the approximate optimization of $C_{S}$ at, say, $N=32$ can be completed in about 5 large-eddy simulations the overhead of SIPI is comparable to a single simulation at an only 1.5 times higher resolution. In addition, the iterands that are collected at $N=32$ provide an appreciation of the sensitivity of simulation results on numerical parameters. Such would be absent when the single-shot simulation at the higher resolution is considered. Further confidence in simulation results at higher resolution would also require the repetition of such simulations at slightly varied model and numerical parameters, i.e., add considerably to the costs. This modest overhead of

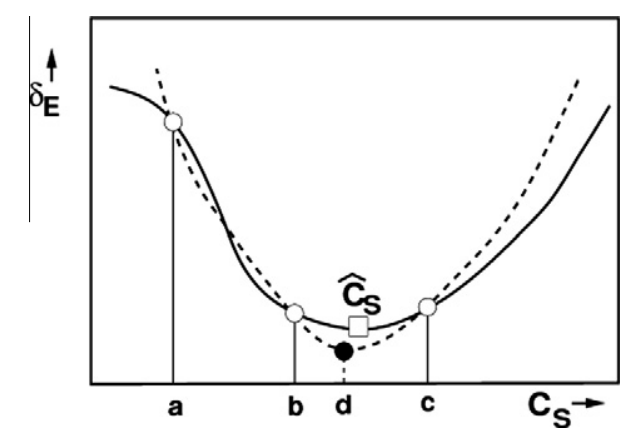

Fig. 2. Illustration of SIPI to approximate the optimal Smagorinsky parameter $\widehat{C}_{S}$ at the ' $\square$ '-symbol where the error-measure $\delta_{E}$ is minimal. The points $(a, b, c)$ define an interpolating polynomial (dashed), whose minimum yields a next approximant $d$ and a new 'bracketing' interval from which to continue the iteration. 
SIPI also comes with a drawback. The choice to minimize errors at fixed $N$ implies that the error can not be reduced to any desired level - if the latter is required then there is no other option than to allow for much higher $N$. In other, practically more restricted settings SIPI can provide a helpful alternative. We will consider examples of this later in the paper.

In the next Section we will introduce the test-case and sketch reference simulation results that are obtained with LES. A systematic error analysis will be discussed in Section 5 .

\section{Experimental and numerical results of bluff-body combustion}

In this section we first identify in some detail the bluff-body combustion test-case that was considered for LES error analysis (Subsection 4.1). Subsequently, we give an overview of simulation results, compared to experimental data in Subsection 4.2.

\subsection{Test case}

The test-case considered in this work is the turbulent non-premixed bluff-body burner designed at Sydney University [33], for which both flow-field and scalar data are available [33,?,35]. The burner features a flow-field of sufficient complexity for a challenging test-case, but avoids unnecessary complications and transient phenomena such as ignition, flame blow-off, or swirl, which may be considered in a future error analysis. The Sydney bluff-body burner consists of an axial bluff-body located in an open wind-tunnel, causing a toroidal air recirculation zone downstream of the bluff-body. A circular hole in the center of the bluff-body allows to eject gaseous fuel into the recirculation zone, leading to a flame that is stabilized by recirculation. The diameter of the bluff-body is $50 \mathrm{~mm}$, the diameter of the fuel injector is $3.6 \mathrm{~mm}$. A set of different flow-rates and fuel-compositions was examined with this burner. The present work focuses on the test-case abreviated HM1e, featuring a coflow velocity of $U_{c o}=35 \mathrm{~m} / \mathrm{s}$, a jet velocity of $U_{j}=108 \mathrm{~m} / \mathrm{s}$, and a fuel composition of $50 \%$ [vol.] natural gas (methane) and 50\% [vol.] hydrogen. For HM1e, the flow is dominated by the air-coflow but the recirculation zone is rich, so that the flame burns in the outer shear layer, at one bluff-body radius. Figure 3 shows a sketch of the burner, together with an instantaneous snapshot of the temperature field as calculated by the LES.

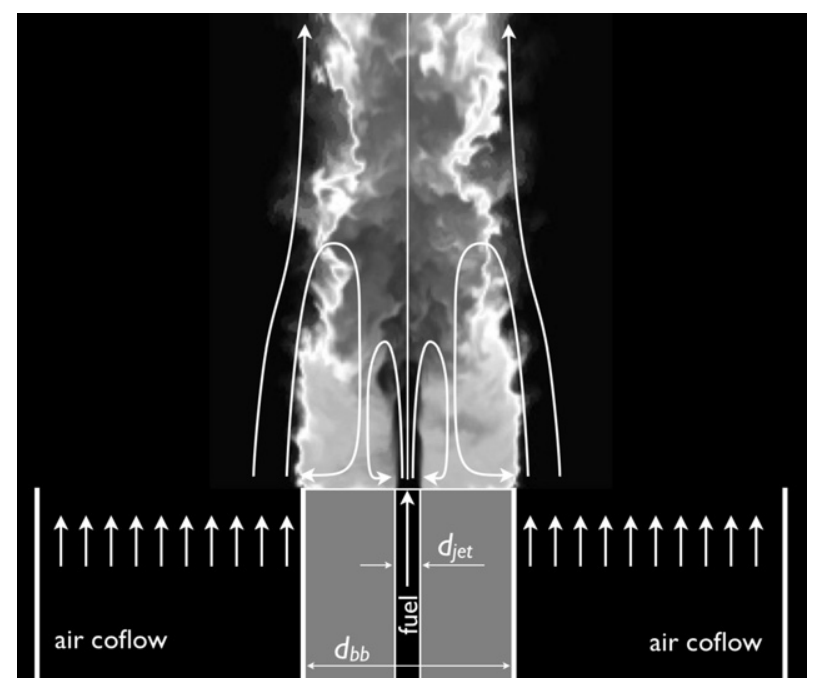

Fig. 3. The Sydney bluff-body burner, with a snapshot of the temperature field predicted by the LES. The calculation was performed with 40 million cells, with a grid-resolution of $0.5 \mathrm{~mm}$. One pixel in the image corresponds to an LES cell. $\left(d_{\text {jet }}=3.6 \mathrm{~mm}, d_{b b}=50 \mathrm{~mm}\right)$.
The burner experiments were performed by Laser Doppler Velocimetry (LDV) [33] and Raman measurements [34,35], which are non-intrusive and cannot alter the flow- and scalar fields in any way. Following an analysis by Veynante and Knikker [36], one would expect that the resulting probe-volumes of the experiment will induce a small amount of instantaneous spatial low-pass filtering, slightly reducing the level of the measured second moments of velocities and scalars. However, it should be noted that even on the finest grid, the LES filter-width remains larger than the experimental probe-volumes, so that the comparison of experiment and simulation will not be unduly affected by the (small) error resulting from the finite probe size.

The Sydney bluff-body flame has been discretised on a computational domain of $200 \mathrm{~mm}$ in axial and $160 \mathrm{~mm}$ in the lateral directions, using cubic cells of $[2.5,2.0,1.5,1.0,0.5] \mathrm{mm}$ length on grids of $[0.63,1.8,5.0,14.1,40.0]$ million cells, where the coarsest grids were chosen to investigate how insufficient resolution affects the error-landscape. Seven simulations were performed on each of these grids for different values of the Smagorinsky parameter $C_{S}$. The simulations were run to a physical time of at least $0.1 \mathrm{~s}$. This time-interval was found to be sufficient for gathering accurate statistics of this combustion problem [38]. In total, generating this error-landscape required almost three CPU years on $2.0 \mathrm{GHz}$ AMD Opteron cores using an MPI communication layer, either via shared memory or over myrinet connections. The largest simulations were run on three quad-core nodes using approximately $10 \mathrm{~GB}$ of memory, whereas the smallest simulations were finished on a single core in less than two hours. We estimate that the total CPU time spent for the creation of the error-landscape (24 thousand hours) would be required for a single spatial resolution of approximately $0.3 \mathrm{~mm}$, requiring almost 200 million cells.

Table 1 provides an overview of all the cases that were run for this work. The computational cost does not evolve according to a simple law, as inter-process communication was added for larger cases; first between processor cores, then between cluster nodes.

\subsection{Comparison of numerical and experimental results for the Sydney bluff-body flame}

This section compares the results of LES and experimental data. The focus of the comparisons in the next section is on the errors in velocity and scalar quantities; here, we first discuss the general behavior of the computational flames at various numerical and model settings to obtain an overview of the flow and scalar fields.

Figures 4-11 show the radial profiles of the mean and fluctuation values of the axial velocity, the radial velocity, the mixture fraction, and the resulting temperature. The plots compare the experimental data to simulation results obtained on five different grids for a Smagorinsky constant of $C_{S}=0.13$, which was obtained from the error-landscape (Fig. 17) as the best possible value. Computational data were presented at experimental measurement points only, making the computed results appear 'less smooth' than the underlying data.

The mean axial velocity plots (Fig. 4) show the high speed of the fuel-jet close to the bluff-body, and also the coflow of air. Further downstream, a recirculation-zone develops at $r / R \approx 0.4$, which remains a prominent flow structure up to a location much further downstream where the jet and coflow merge. The mean radial velocity is presented in Fig. 5, providing more information on the recirculation zone. The mean radial velocity is relatively hard to measure and to predict, as its magnitude is very small compared to the axial velocity. This leads to a large relative error in the mean radial velocity, even though it's absolute error is an order of magnitude smaller than that of the axial velocity.

The axial velocity fluctuation is shown in Fig. 6, with peaks at $r / R \approx 0.05$ and $r / R \approx 1.0$, indicating the shear layers around the 
Table 1

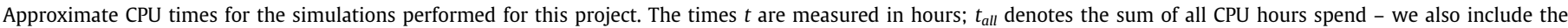
fraction $t / t_{\text {all }}$ used by simulations at a given resolution. The reference Smagorinsky value is the Lilly proposal $C_{S 0}=0.173$.

\begin{tabular}{|c|c|c|c|c|c|c|c|c|c|c|}
\hline $\begin{array}{l}\text { Cells } \\
\left(10^{6}\right)\end{array}$ & $\begin{array}{l}\Delta \\
(\mathrm{mm})\end{array}$ & $\begin{array}{l}\frac{1}{4} C_{S 0} \\
(t / \mathrm{h})\end{array}$ & $\begin{array}{l}\frac{1}{2} C_{S 0} \\
(t / \mathrm{h})\end{array}$ & $\begin{array}{l}\frac{3}{4} C_{S 0} \\
(t / \mathrm{h})\end{array}$ & $\begin{array}{l}C_{S 0} \\
(t / h)\end{array}$ & $\begin{array}{l}\frac{5}{4} C_{S 0} \\
(t / h)\end{array}$ & $\begin{array}{l}\frac{6}{4} C_{S 0} \\
(t / h)\end{array}$ & $\begin{array}{l}2 C_{S 0} \\
(t / h)\end{array}$ & $t / t_{\text {all }}$ & $\begin{array}{l}\text { CPU } \\
\text { (cores) }\end{array}$ \\
\hline 0.3 & 2.5 & 1.5 & 1.5 & 1.5 & 1.5 & 1.5 & 1.5 & 1.5 & $4 \times 10^{-4}$ & 1 \\
\hline 0.6 & 2.0 & 6 & 6 & 6 & 6 & 6 & 6 & 6 & $1.8 \times 10^{-3}$ & 1 \\
\hline 1.5 & 1.5 & 28 & 28 & 28 & 28 & 28 & 28 & 28 & $8.0 \times 10^{-2}$ & 4 \\
\hline 5.0 & 1.0 & 150 & 150 & 150 & 150 & 150 & 150 & 150 & 0.04 & 4 \\
\hline \multirow[t]{2}{*}{40.0} & 0.5 & 3200 & 3200 & 3200 & 3200 & 3200 & 3200 & 3200 & 0.94 & $3 \times 4$ \\
\hline & $t / \mathrm{h}$ & 3400 & 3400 & 3400 & 3400 & 3400 & 3400 & 3400 & 2.7 years & \\
\hline
\end{tabular}
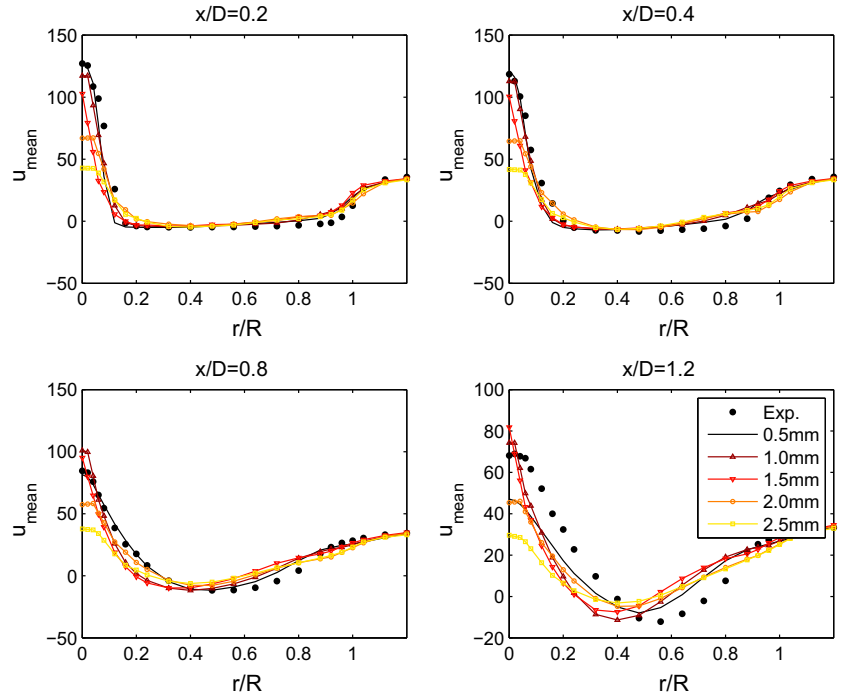

Fig. 4. Mean axial velocity for different grid resolutions at $C_{S}=0.13$.
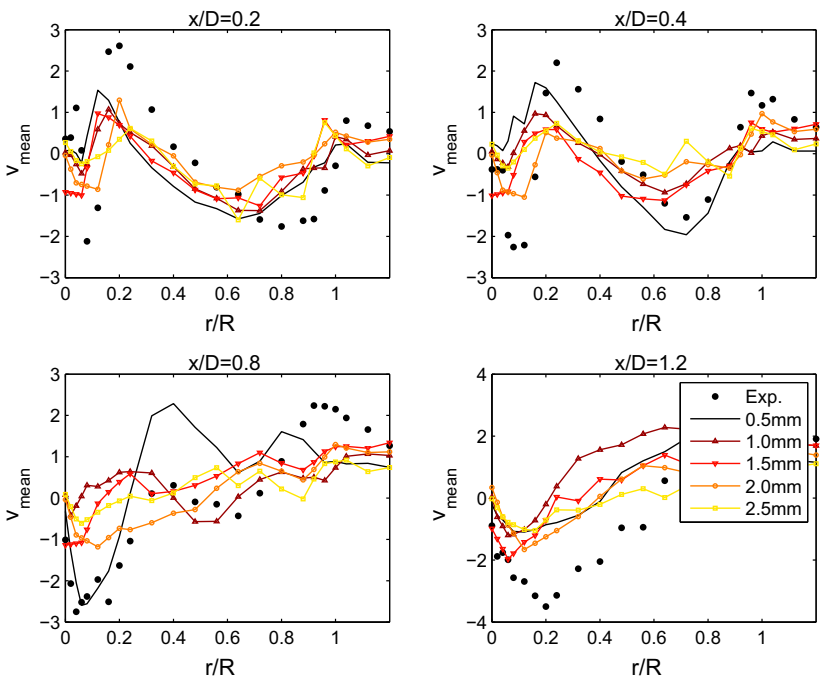

Fig. 5. Mean radial velocity for different grid resolutions at $C_{S}=0.13$.

central jet and on the inside of the coflow. The radial velocity fluctuations presented in Fig. 7 behave very similar, and for both fluctuation levels, a sufficient grid-resolution is required to obtain a good prediction.

Figure 8 shows the mixture fraction profile across the diameter of the flame, with measurements taken on two sides of the centerline. The plots show a rich recirculation zone downstream of the bluff-body, and the pure fuel that is ejected from the nozzle. The
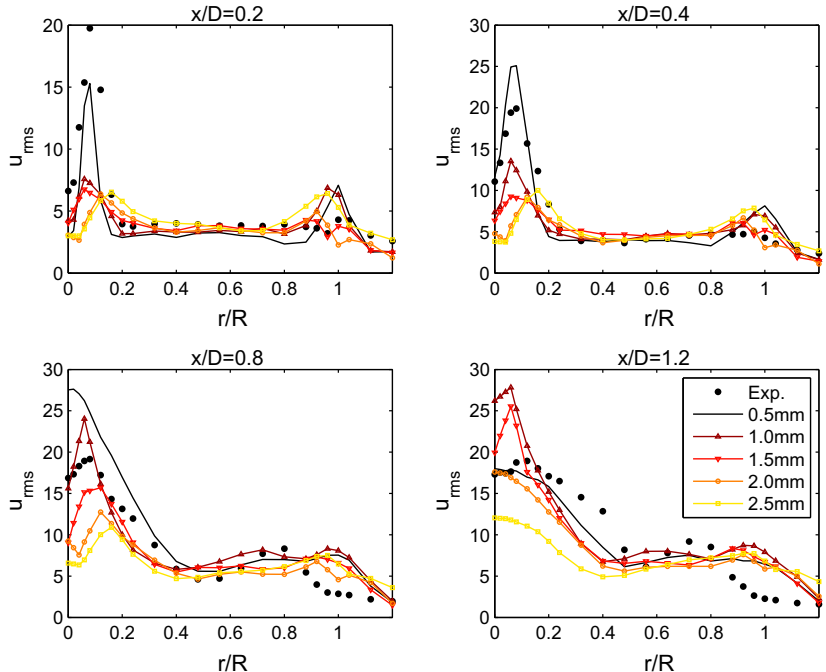

Fig. 6. Fluctuation of axial velocity for different grid resolutions, $C_{S}=0.13$.
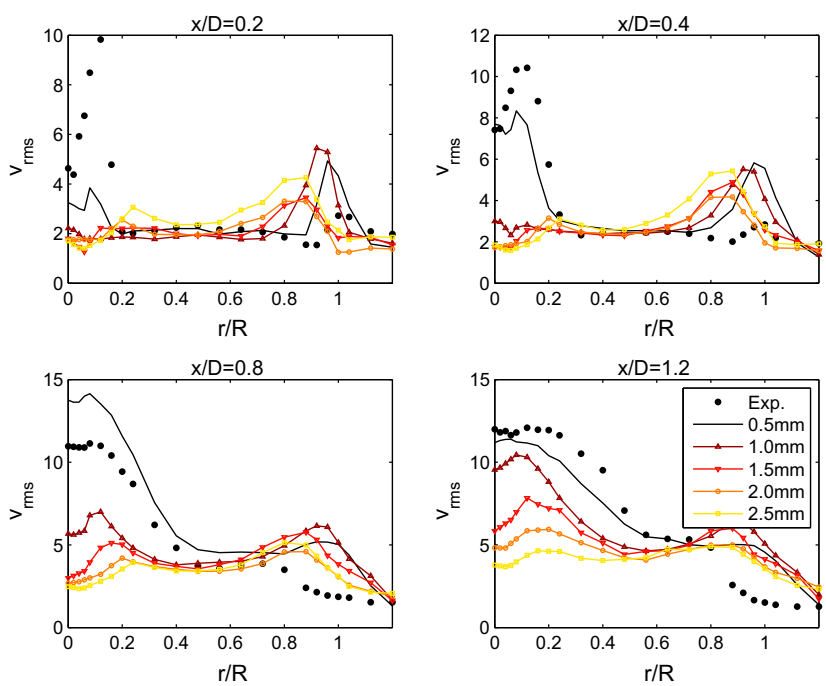

Fig. 7. Fluctuation of radial velocity for different grid resolutions, $C_{S}=0.13$.

mean temperature field is a direct result of the unsteady mixture fraction fields, and is presented in Fig. 9. The mixture fraction fluctuations are shown in Fig. 10; the mixture fraction fluctuations are relatively high on the coarse grids while the velocity fluctuations were found quite small on these grids. These deviations may be explained by the fact that on the coarse grid, the flame is generally predicted too short, so that radial profiles are shifted upstream. Finally, Fig. 11 shows the temperature fluctuations, which behaves as expected from the mean mixture fraction and its fluctuation. 

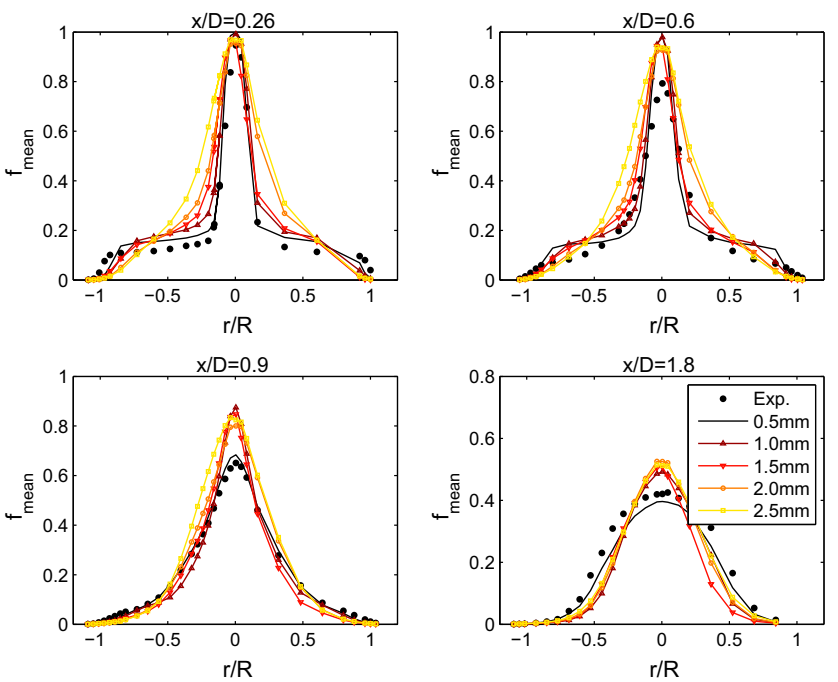

Fig. 8. Mean mixture fraction for different grid resolutions at $C_{S}=0.13$.
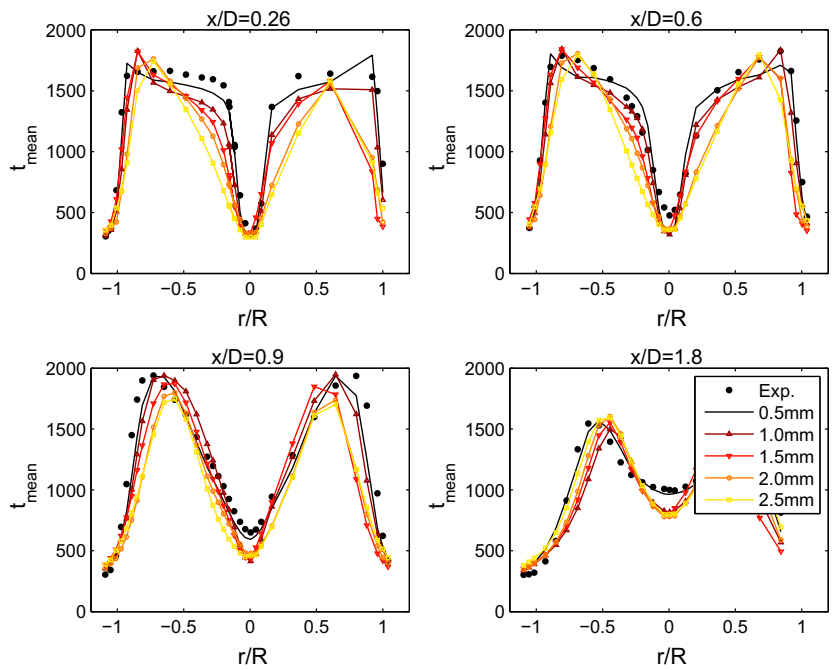

Fig. 9. Mean temperature for different grid resolutions at $C_{S}=0.13$.
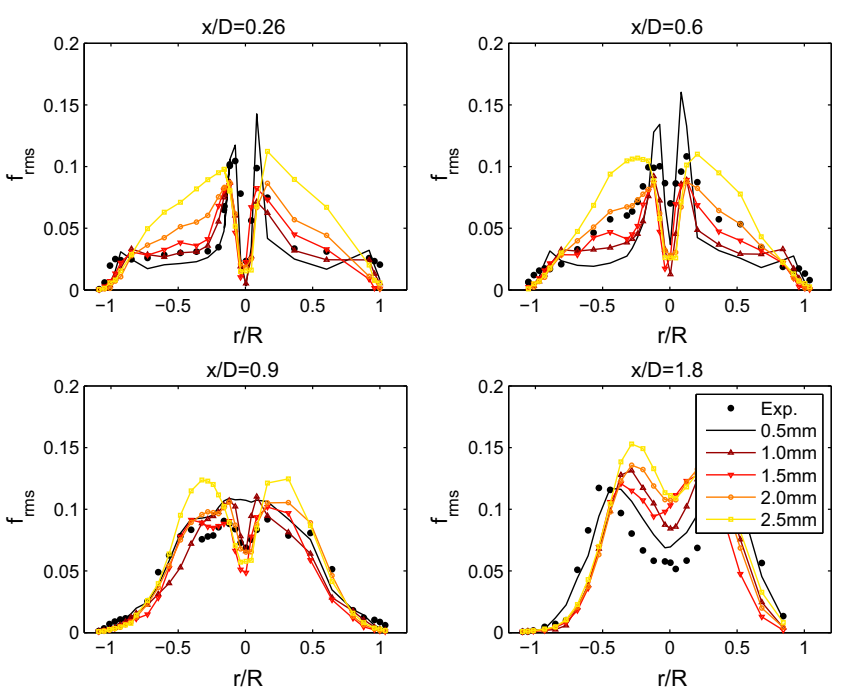

Fig. 10. Fluctuation of mixture fraction for different grid resolutions for $C_{S}=0.13$.
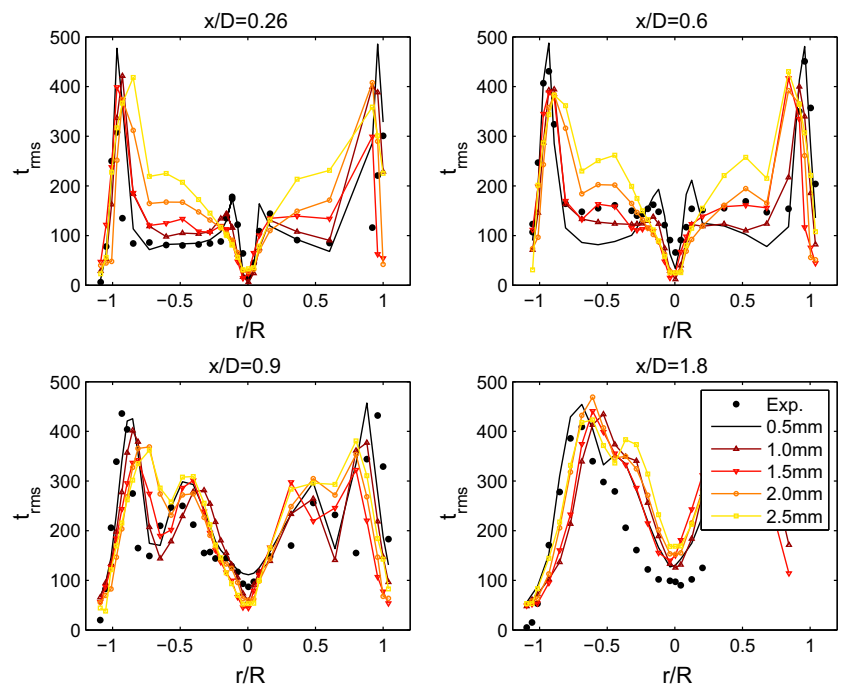

Fig. 11. Fluctuation of temperature for different grid resolutions, $C_{S}=0.13$.

\section{Error analysis of the Sydney flame}

In this section we present an error-analysis based on the systematic recording of so-called error-landscapes of several key properties of the Sydney flame. The error-landscapes show the error between all of the experimental data for certain quantities, such as velocity, temperature and mixture fractions, and the corresponding numerical data interpolated at the same point, as defined in Eq. (6).

Figure 14 shows the error-landscape for the mean axial and radial velocities and their fluctuations $u_{r m s}, v_{r m s}$. The error-landscape for the mean axial velocity shows that the choice of an appropriate model constant combined with a fine grid can reduce the error from approximately $25 \mathrm{~m} / \mathrm{s}$ to a much lower level of $5 \mathrm{~m} / \mathrm{s}$. The landscape looks remarkably similar to the landscape shown by Meyers et al. [25] for the dissipation rate in isotropic, homogenous turbulence, showing the same, potentially generic, valley-like structure. It features the expected asymptotic behavior of a decreasing error with higher spatial resolution, and an increasing error for both low and high values of the Smagorinsky constant $C_{S}$. The stabilizing effect of the Smagorinsky model may also be implied by the $u_{\text {mean }}$ error-landscape, where large $C_{S}$ values lead to a very smooth error-field, whereas small $C_{S}$ values seem to result in rather inconsistent fluctuations in the error-field. This is in-line with the expectation that too high values of the model constant may increase the model-error because of deficiencies in the representation of the sub-filter turbulence, whereas too low values for the model constant can lead to larger numerical errors, which display strong variations with numerical resolution.

The interpretation of the error-landscape becomes more challenging for the fluctuations of the axial velocity $u_{r m s}$. Here again, errors become large for very low $C_{S}$ and for large $C_{S}$ on coarse grids, and the error becomes smallest for the finest grid. However, there appear to be two further band-like low-error areas. The first one occurs for approximately constant $C_{S} \approx 0.14$, the second one for large $C_{S}$ on relatively coarse grids. The latter can be seen in Fig. 12, where relatively high fluctuations are predicted on the $\Delta=1.5 \mathrm{~mm}$ grid, which leads to a small total error as fluctuations are generally under-predicted for $C_{S}=0.346$. This is an illustration of the partial compensation between errors that can arise under certain settings of numerical and modeling parameters.

Figure 14 also shows the error-landscape for the mean radial velocity $v_{\text {mean }}$. The error is small on the finest grid for $C_{S} \approx 0.13$, 

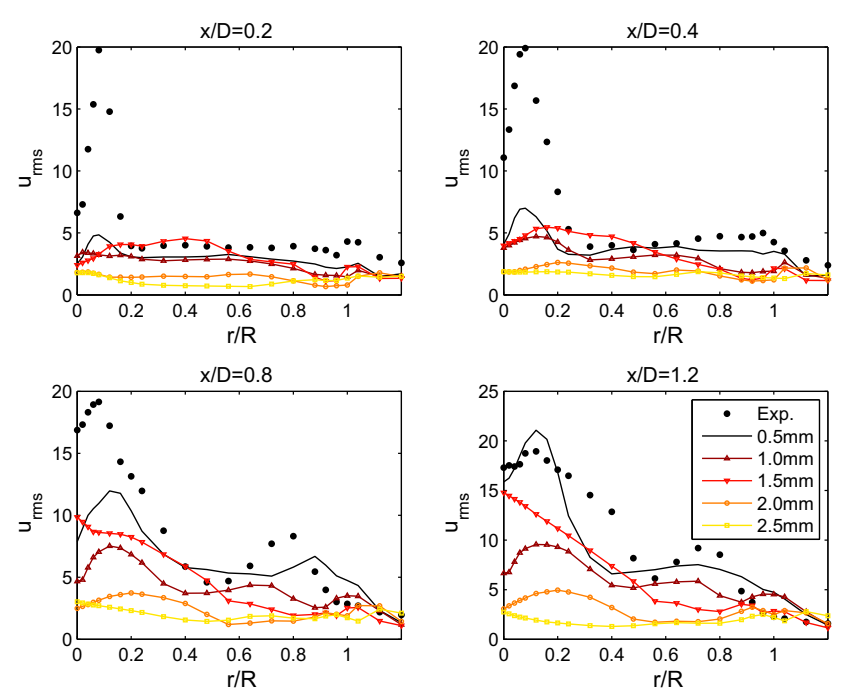

Fig. 12. Fluctuation of axial velocity for different grid resolutions with $C_{S}=0.346$. This very large Smagorinsky constant causes a very high turbulent viscosity, and too much dampening of most oscillations.

and becomes larger for values of $C_{S}$ that are either much lower or much higher, and on coarser grids. There are also low-error islands, for example at $C_{S} \approx 0.13$ and $\Delta \approx 0.15 \mathrm{~mm}$. Figure 7 shows the underlying mean radial velocity data, which is generally hard to predict as the absolute values are very small compared to the axial velocity. The simulations on the coarse grid are affected by numerical oscillation, which are even more obvious in Fig. 13, showing the mean radial velocity for a very low Smagorinsky constant of $C_{S}=0.04$. In general, the error-landscape of the mean radial velocity is rather 'spiky', but it appears to become smoother for large $C_{S}$ and finer grids - as would be required to obtain convergence. However, it must be stressed that the absolute errors in $v_{\text {mean }}$ are approximately ten times lower than in the axial velocity $u_{\text {mean }}$.

Finally, the error in the radial velocity fluctuations $v_{r m s}$ is shown in Fig. 14. Overall, the error for this quantity appears most similar to the error-landscape for energy dissipation presented by Meyers et al. [25]. Interestingly, the error-landscape for the velocity fluctuation $v_{\text {rms }}$ looks much smoother than for the mean velocity $v_{\text {mean }}$.
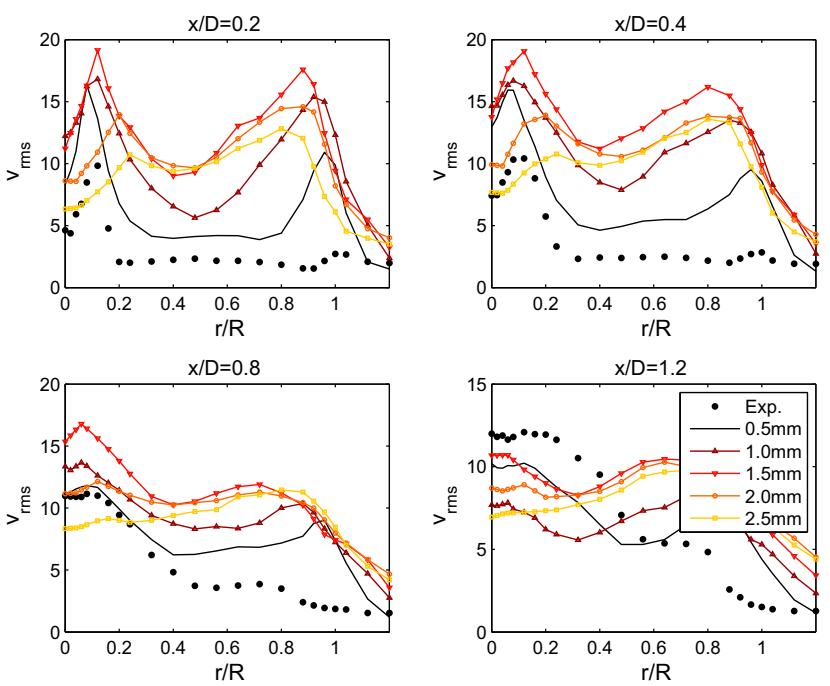

Fig. 13. Fluctuation of radial velocity for different grid resolutions for a very low Smagorinsky constant of $C_{s}=0.04$. The very low resulting turbulent viscosities lead to very high fluctuation levels.
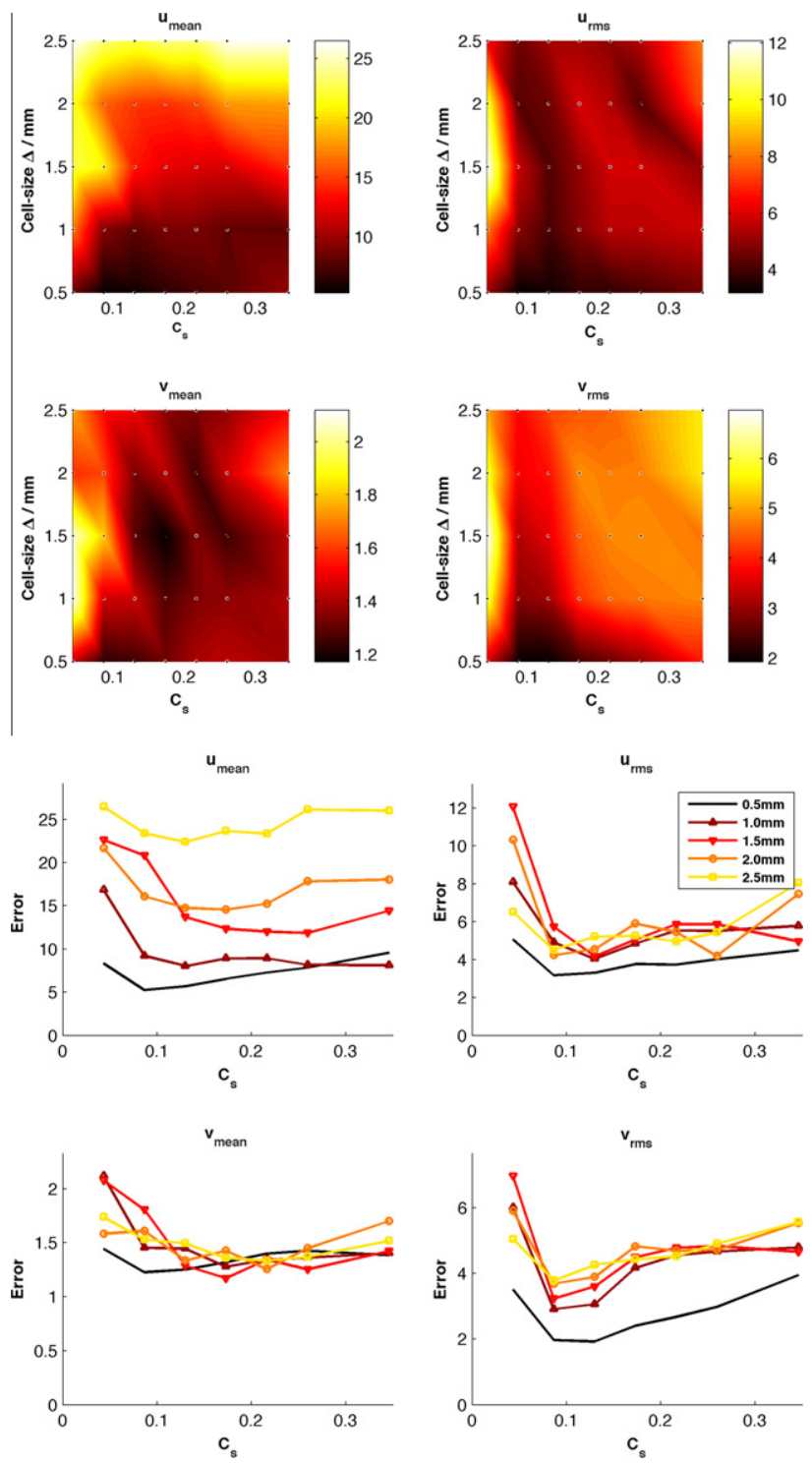

Fig. 14. Error landscape for mean axial and radial velocities $u_{\text {mean }}, v_{\text {mean }}$ and their standard deviations $u_{r m s}, v_{r m s}$. The blue lines show trajectories in negative gradient direction towards lower errors. (Data for this plot is only known for the marker-points, the space inbetween was filled by linear interpolation.) (For interpretation of the references to color in this figure legend, the reader is referred to the web version of this article.)

The error can be minimized by choosing the 'optimum' $C_{S}$ value; the sensitivity to which drops with grid-refinement as in this situation also the filter-width is reduced and the dynamic relevance of the sub-filter turbulence is less pronounced. Figure 15 shows the error-landscape for the means and fluctuations of mixture fraction, and of the temperature field that is derived from it. The error-landscape for the mean mixture fraction shows a clear optimum on the fine grid for $C_{S} \approx 0.13$, where the error gets as low as $5 \%$. Overall, the error-landscape for the mean mixture fraction $f_{\text {mean }}$ is very smooth, not only for large model constants but even for small ones. This may be explained by the use of non-oscillatory TVD schemes for mixture fraction transport, which add numerical diffusion not dissimilar to the diffusion resulting from setting a large $C_{S}$ value [57]. The error for the mixture fraction fluctuation is generally low, with a minimum at $C_{S} \approx 0.13$ and a very distinctive maximum for $C_{S} \approx 0.24$. This peak in the error is caused by a very strong overprediction of the mixture fraction fluctuation, which becomes 

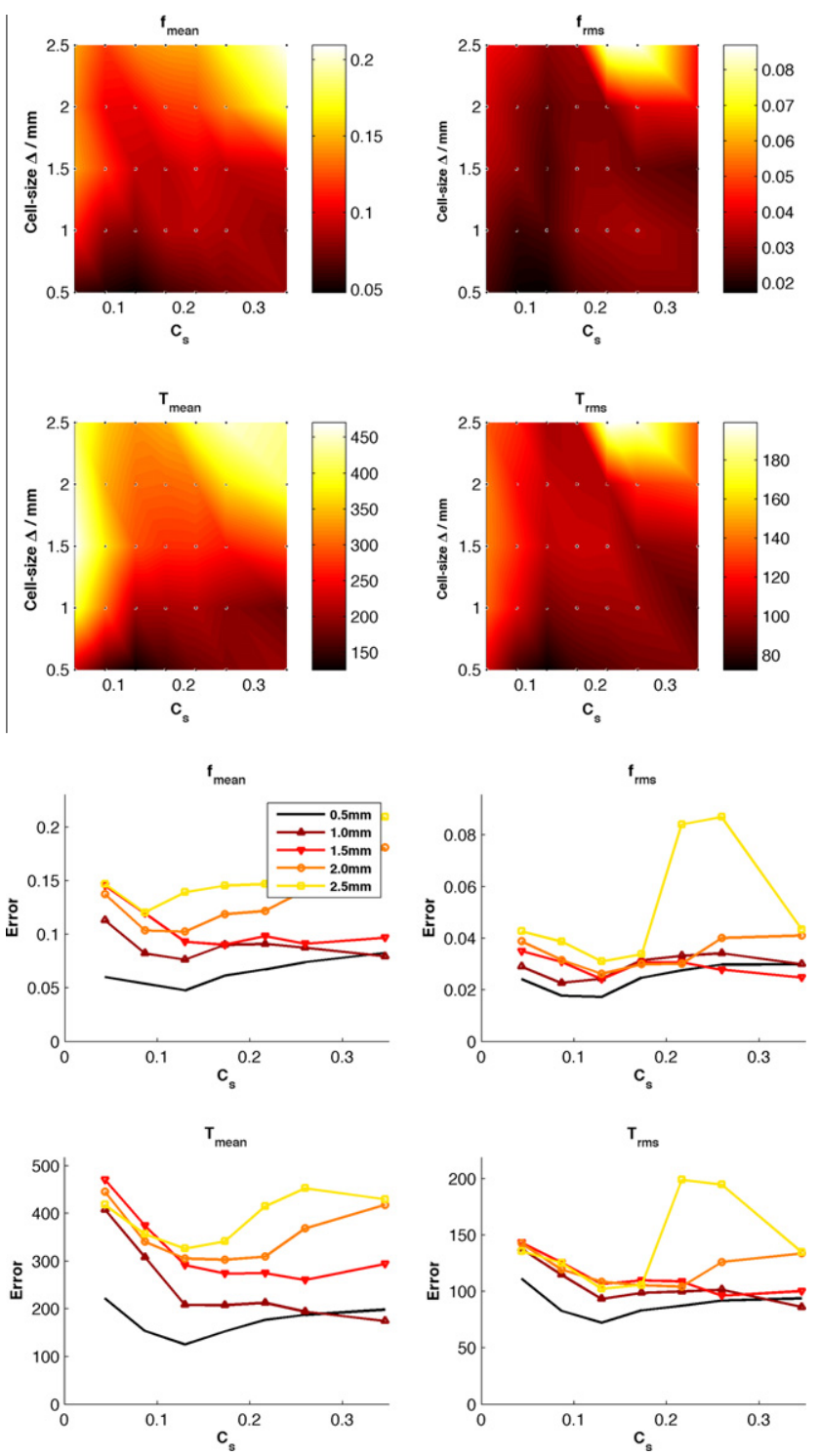

Fig. 15. Error landscape for mixture fraction (f) and temperature (t) mean values and fluctuations. The large error in the fluctuations for $\Delta=2.5 \mathrm{~mm}$ and $C_{S} \approx 0.24$ stems from a puffing motion of the rich recirculation zone, that has only been observed for these parameters. The blue lines show trajectories in negative gradient direction towards lower errors. (Data for this plot is only known for the marker-points, the space inbetween was filled by linear interpolation.) (For interpretation of the references to color in this figure legend, the reader is referred to the web version of this article.)

strikingly obvious in Fig. 16 where we show results for $C_{S}=0.216$. This over-prediction only occurs on the coarsest grid, for a Smagorinsky constant around $C_{S}=0.24$. Looking at time-resolving animations of the predicted mixture fraction fields, one observes a puffing motion in the length of the recirculation zone. This oscillation is not observed in the other simulations, and can explain the very high predicted mixture fraction fluctuation. This instability is not observed in the experiment; it illustrates the extent to which an inappropriate grid and model constant can alter the perception of the flow-physics, supporting further the need for parameter studies and more research into methods ensuring LES quality. Finally, Fig. 15 shows the error-landscapes for the temperature mean and its fluctuation. The present simulations are based on a steady flamelet assumption, which implies that the temperature and concentration fields are all non-linear functions of the instantaneous mixture fraction field. The error-landscapes for the temperature
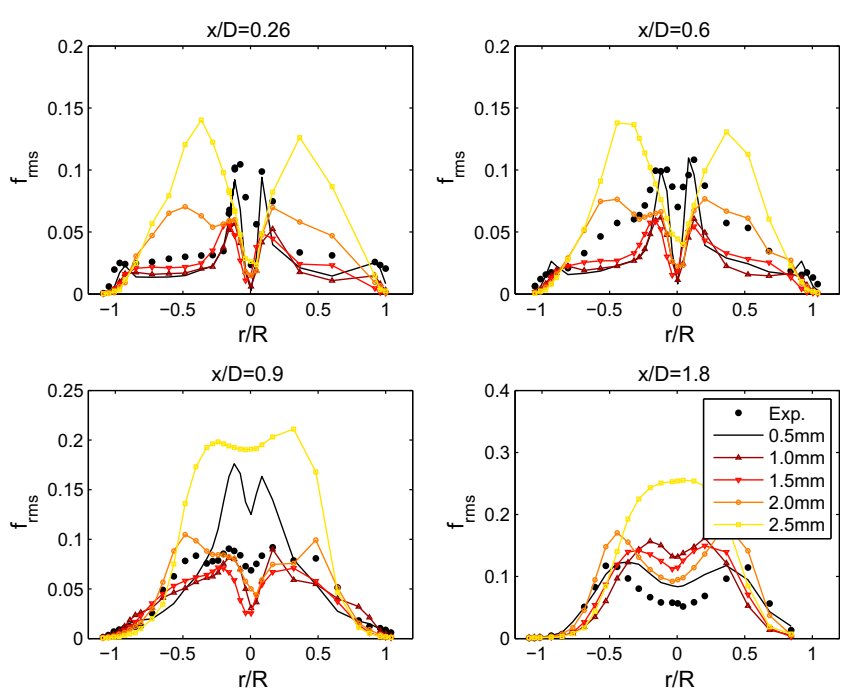

Fig. 16. Fluctuation of mixture fraction for different grid resolutions at $C_{s}=0.216$. Interestingly, a strong puffing motion was observed on the coarsest grid ( $\Delta=2.5 \mathrm{~mm}$, yellow line) that leads to very large fluctuation levels. The same puffing was observed for $C_{s}=0.26$, but in no other case. (For interpretation of the references to color in this figure legend, the reader is referred to the web version of this article.)

hence do not provide any significant information beyond that already obtained from the mixture fraction field. Overall, the error in the mean temperature can be reduced to approximately $100 \mathrm{~K}$. This level of temperature error corresponds to about 5\% error and is mainly due to a spatial shift of regions with very strong temperature gradients.

Error landscapes for the first and second moments of velocity, mixture fraction and temperature can be combined to obtain a better impression of the quality of a simulation. We are interested in the topography of the error-landscape of the total error and combine the separate error-landscapes of specific quantities after normalization by the respective maxima. Fig. 17 shows the global error landscape. A minimum-error valley exists, but is not as distinct and well-defined as in Meyers et al. [25] study of isotropic decaying turbulence. This suggests that finding an optimal value of the Smagorinsky constant is less critical in the present case of a complicated turbulent reactive flow. However, this perceived insensitivity could also be due to the existence of other interacting errors that will dominate if the Smagorinsky constant gets small. In the case presented, such errors may be due to inaccuracies in the computational boundary conditions relative to the experiment, or due to experimental errors that are not negligible. A further explanation for the low-error valley being less distinct in the present simulation may be that the bluff-body flame includes many different fluid-mechanical features, with very different local resolution
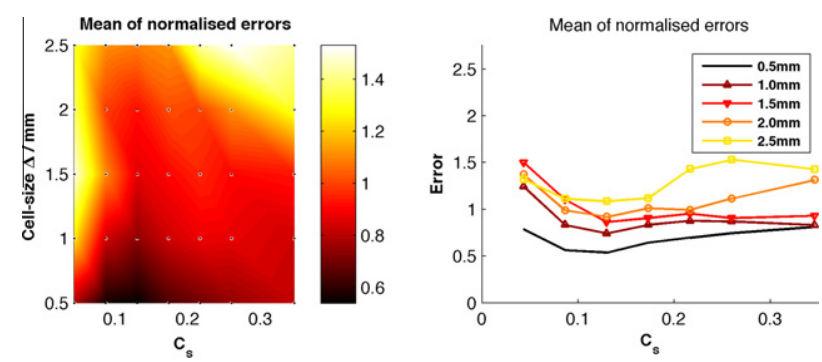

Fig. 17. Global error-landscape calculated as the mean of the normalized individual error-landscapes. (Data for this plot is only known for the marker-points, the space inbetween was filled by linear interpolation.) 
requirements. This would ideally be represented by the dynamic modeling procedure, which provides locally varying Smagorinsky coefficients in both space and time. Future efforts on this topic will include use of dynamic modeling to further investigate these observations.

It should also be stressed that typical engineering LES will use dynamic modeling in conjunction with locally refined, possibly unstructured grids, so that further work will be required to assess the suitability of the error-landscape approach for such simulations.

\section{Conclusions and summary}

We have performed a systematic study of the effect of the grid resolution and the Smagorinsky constant on large-eddy simulations of the non-premixed Sydney flame. Results of the LES were compared to experimental data. Errors in the predicted first and second moments of velocities, mixture fraction, and temperature were calculated at over 100 points in physical space, from which a single mean error was computed for each quantity and each simulation. For each quantity, the mean error was plotted versus the grid-resolution and the Smagorinsky constant. The data were compiled using the concept of an error-landscape to provide an overview of the total simulation error at various modeling and numerical settings. In particular, near-optimal values for the Smagorinsky constant were identified over a range of grid resolutions. The error-landscape map provides an indication of where the combination of competing model and numerical errors are simultaneously minimized, and to what extent.

Analysis of the error-landscapes indicated that a global minimum existed for the finest grid, at a Smagorinsky constant of approximately 0.13 . For coarser grid resolutions, the minimumerror in most quantities was located on a line of an almost constant Smagorinsky parameter of 0.13 , showing a similar valley-structure as observed in the analysis of homogeneous decaying turbulence. The present work shows that the concept of an error-landscape introduced by Meyers et al. [25] can be extended for compressible, anisotropic turbulence in complex geometries. For most quantities, the resulting landscapes are reminiscent to those for homogeneous decaying turbulence, showing a steep increment in error for very low Smagorinsky constants where the numerical error starts to dominate. The error was found to increase less rapidly for Smagorinsky coefficients that are larger than the optimum. As expected, the error is reduced with finer grid resolution. Such error-analyses add the important quantification of sensitivity of the simulation results to variations in the parameters.

The error-field was found to be smooth for large model coefficients and fine grids. The 'roughness' of the error-landscape may indicate regions where the Smagorinsky coefficient was too small. This roughness may indicate simulation quality. In cases where experimental data is available and the error-landscape is smooth, an optimal value for the Smagorinsky constant can effectively be determined using the SIPI procedure $[55,29]$. This has important implications regarding the efficiency of the method.

The error-landscape approach can help identify combinations of grid-resolution and model constant that may induce qualitatively wrong flow and combustion physics into the calculations. This problem is illustrated by the results on the coarsest grid with model constants around 0.24 , for which an unrealistic puffing mode of the flame leads to excessive fluctuations. This observation was easily made by correlating qualitative observations of animations to the quantative results seen in the error-landscape for the mixture fraction fluctuations.

A main pacing item in the research on reliability of large-eddy simulation is to find error-measures that can be assessed on the basis of basic fluid-mechanical considerations and processing of

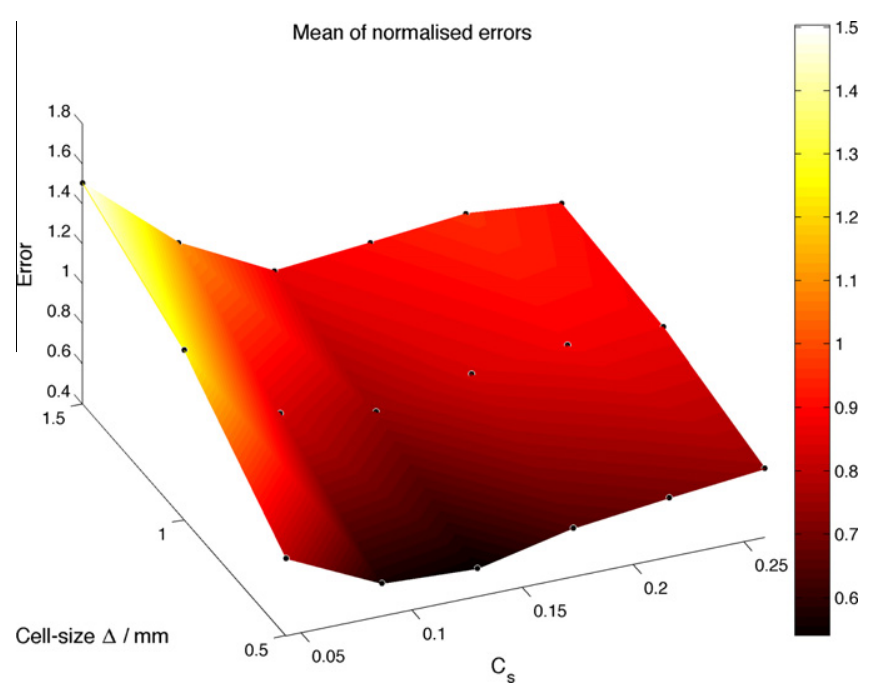

Fig. 18. Global error-landscape calculated as the mean of the normalized individual error-landscapes. In this picture, the coarsest grids resolutions and the highest Smagorinsky constant have been discarded to present the smooth region of the error-landscape in a similar style as used by Meyers et al. [25].

numerical results alone. Current work on computational erroranalysis was restricted thus far to further the understanding of interacting errors due to numerical and modeling deficiencies. Research will be directed toward a self-contained error measure for large-eddy simulation in the future Fig. 18.

\section{Acknowledgments}

The authors would like to acknowledge the support of the UK Engineering and Physical Sciences Research Council (EPSRC), and also the US Department of Energy, Office of Basic Energy Sciences, Division of Chemical Sciences, Geosciences, and Biosciences. We are grateful to Rob Barlow at Sandia National Laboratories, the main organiser of the TNF Workshop from which the present paper has evolved.

\section{References}

[1] J. Smagorinsky, Mon. Weather Rev. 91 (1963) 99-164.

[2] P. Sagaut, Large Eddy Simulation for Incompressible Flow; An Introduction, Scientific Computation, Springer-Verlag, 2001.

[3] B.J. Geurts, Elements of direct and large-eddy simulation, R.T. Edwards, Inc, 2003.

[4] T. Poinsot, D. Veynante, Theoretical and Numberical Combustion, R.T. Edwards, Inc., 2005.

[5] A. Cook, J. Riley, Phys. Fluids 6 (8) (1994) 2868-2870.

[6] N. Branley, W.P. Jones, Large eddy simulation of a turbulent non-premixed flame, in: Proceedings of the Eleventh Symposium on Turbulent Shear Flows, Grenoble, France, 1997, pp. 21-1-21-6.

[7] H. Forkel, J. Janicka, Flow, Turb. Combust. 65 (2000) 163-175.

[8] A. Kempf, A. Sadiki, J. Janicka, Proc. Combust. Inst. 29 (2002) 1979-1985.

[9] F.A. Williams, Recent advances in theoretical descriptions of turbulent diffusion flames, in: S.N.B. Murthy (Ed.), Turbulent Mixing in Nonreactive and Reactive Flows, Plenum Press, New York, 1975.

[10] N. Peters, Prog. Energy Combust. Sci. 10 (1984) 319-339.

[11] H. Pitsch, Proc. Combust. Inst. 29 (2002) 1971-1978.

[12] S. Navarro-Martinez, A. Kronenburg, Proc. Combust. Inst. 32 (2009) 15091516.

[13] A. Klimenko, R.W. Bilger, Progr. Energy Combust. Sci. 25 (1999) 595-687.

[14] H. Steiner, W. Bushe, Phys. Fluids 13 (2001) 754-769.

[15] P.J. Colucci, F.A. Jaberi, P. Givi, S.B. Pope, Phys. Fluids 10 (2) (1998) 499-515.

[16] A. Kerstein, J. Fluid Mech. 240 (1992) 289-313.

[17] W. Calhoon, S. Menon, AIAA Paper 96-0516 (1996).

[18] V. Raman, H. Pitsch, Proc. Combust. Inst. 31 (2007) 1711-1719.

[19] F. Bisetti, J.-Y. Chen, LES of Sandia flame D with Eulerian PDF and finite-rate chemistry, 2005 Fall Meeting Western States Combustion Institute, Stanford, CA, October 17-18, 2005. Paper 05F-33. http://repositories.cdlib.org/cpl/cm/ BisettiWSSF05.

[20] R. Mustata, L. Valino, C. Jimenez, S. Bondi, Combust. Flame 145 (2006) 88-104. 
[21] L. Vervisch, T. Poinsot, Ann. Rev. Fluid Mech. 30 (1998) 655-692.

[22] J. Janicka, A. Sadiki, Proc. Combut. Inst. 30 (2005) 537-547.

[23] J.C. Oefelein, Prog. Aero. Sci. 42 (2006) 2-37.

[24] H. Pitsch, Ann. Rev. Fluid Mech. 38 (2006) 453-482.

[25] J. Meyers, B.J. Geurts, M. Baelmans, Phys. Fluids 15 (2003) 2740-2755.

[26] J. Meyers, B.J. Geurts, M. Baelmans, Phys. Fluids 17 (2005) 045108

[27] J. Meyers, P. Sagaut, B.J. Geurts, Phys. Fluids 18 (2006) 095103.

[28] A.W. Vreman, B.J. Geurts, J.G.M. Kuerten, Int. J. Numer. Methods Fluids 22 (1996) 299-311.

[29] B.J. Geurts, J. Meyers, Phys. Fluids 18 (2006) 118102.

[30] S. Ghosal, J. Comput. Phys. 125 (1996) 187-206.

[31] A.W. Vreman, B.J. Geurts, J.G.M. Kuerten, Commun. Numer. Methods Eng. Math. 10 (1994) 785-790.

[32] B.J. Geurts, J. Fröhlich, Phys. Fluids 14 (2002) L41.

[33] B. Dally, D. Fletcher, A. Masri, Combust. Theory Model. 2 (1998) 193-219.

[34] A. Masri, B. Dally, R. Barlow, C. Carter, Proc. Combust. Inst. 25 (1994) 1301-1308.

[35] B. Dally, A. Masri, R. Barlow, G. Fiechtner, Combust. Flame 114 (1998) 119-148.

[36] D. Veynante, R. Knikker, J. Turb. 7 (2006) Article 35.

[37] A.W. Vreman, B.J. Geurts, J.G.M. Kuerten, Appl. Scient. Res. 54 (1995) 191-203.

[38] A. Kempf, R.P. Lindstedt, J. Janicka, Combust. Flame 144 (2006) 170-189.

[39] V.Sick, F. Hildenbrand, R.P. Lindstedt, Proc. Combust. Inst. 27 (1998) 1401-1409

[40] U. Schumann, R. Sweet, J. Comput. Phys. 20 (1976) 171-182.

[41] U. Piomelli, J. Ferziger, P. Moin, J. Kim, Phys. Fluids A 1 (6) (1989) 1061-1068

[42] D.K. Lilly, The representation of small-scale turbulence in numerical simulation experiments, in: Proceedings of IBM Scientific Computing Symposium on Environmental Sciences, 1967, pp. 195-210.
[43] M. Germano, U. Piomelli, P. Moin, W.H. Cabot, Phys. Fluids 3 (1991) 17601765.

[44] D.K. Lilly, Phys. Fluids 4 (1992) 633-635.

[45] B.J. Geurts, Reliability of LES in complex applications, in: Advances in Hybrid RANS-LES Modelling, Springer, 2008. pp. 10-20.

[46] R. Bilger, S. Starner, R. Kee, Combust. Flame 80 (1990) 135-149.

[47] J. Floyd, A.M. Kempf, A. Kronenburg, H.R. Ram, Combust. Theory Model. 13 (4) (2009) 559-588.

[48] C. Olbricht, J. Janicka, A. Kempf, LES as a prediction tool for lifted flames in a model gas turbine combustor, in: Proceedings of ASME Turbo Expo 2010, June 14-18, 2010, Glasgow, UK, 2010, paper GT2010-22525.

[49] M.W.A. Pettit, B. Coriton, A. Gomez, A.M. Kempf, Proc. Combust. Inst. 33 (2011) 1391-1399.

[50] O. Stein, A. Kempf, Proc. Combust. Inst. 31 (2007) 1755-1763.

[51] A.M. Kempf, W. Malalasekera, K.K.J. Ranga-Dinesh, O. Stein, Flow Turb. Combust. 81 (2008) 523-561.

[52] A. Kempf, M. Klein, J. Janicka, Flow Turb. Combust. 74 (2005) 67-84.

[53] A.M. Kempf, Flow Turb. Combust. 80 (2008) 351-373.

[54] B. Böhm, J. Brübach, C. Ertem, A. Dreizler, Flow Turb. Combust. 80 (2008) 507529.

[55] R. Brent, Algorithms for Minimization without Derivatives,;, Englewood Cliffs N.J., Prentice-Hall, 1973.

[56] U. Piomelli, B.J. Geurts, A grid-independent length scale for large-eddy simulations, J. American Physical Society, 62nd Annual Meeting of the APS Division of Fluid Dynamics, November 22-24, 2009, paper \#EB.003.

[57] B.J. Geurts, F. van der Bos, Phys. Fluids 17 (2005) 125103. 